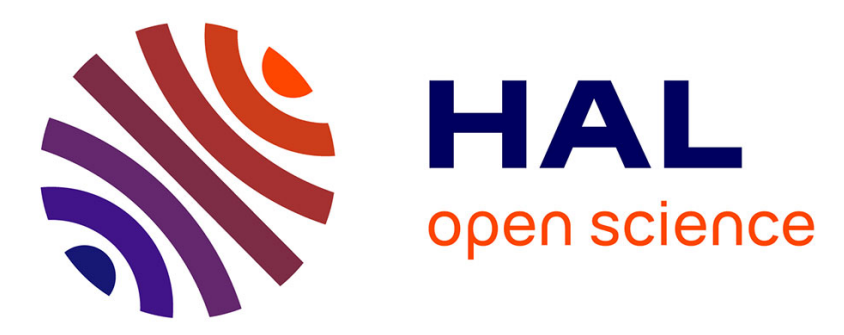

\title{
GROUND AMPLIFICATION IN THE NEIGHBORHOOD OF A GROUP OF MUTUALLY PARALLEL TRIANGULAR RIDGE MOUNTAINS SUBJECTED TO SHEAR HORIZONTAL SEISMIC WAVES
}

\author{
A. Wirgin, L. Kouoh-Bille
}

\section{To cite this version:}

A. Wirgin, L. Kouoh-Bille. GROUND AMPLIFICATION IN THE NEIGHBORHOOD OF A GROUP OF MUTUALLY PARALLEL TRIANGULAR RIDGE MOUNTAINS SUBJECTED TO SHEAR HORIZONTAL SEISMIC WAVES. Journal de Physique IV Proceedings, 1992, 02 (C1), pp.C1-729C1-732. 10.1051/jp4:19921158 . jpa-00251117

HAL Id: jpa-00251117 https://hal.science/jpa-00251117

Submitted on 1 Jan 1992

HAL is a multi-disciplinary open access archive for the deposit and dissemination of scientific research documents, whether they are published or not. The documents may come from teaching and research institutions in France or abroad, or from public or private research centers.
L'archive ouverte pluridisciplinaire HAL, est destinée au dépôt et à la diffusion de documents scientifiques de niveau recherche, publiés ou non, émanant des établissements d'enseignement et de recherche français ou étrangers, des laboratoires publics ou privés. 


\title{
GROUND AMPLIFICATION IN THE NEIGHBORHOOD OF A GROUP OF MUTUALLY PARALLEL TRIANGULAR RIDGE MOUNTAINS SUBJECTED TO SHEAR HORIZONTAL SEISMIC WAVES
}

\author{
A. WIRGIN and L. KOUOH-BILLE
}

Laboratoire de Modélisation en Mécanique, U.R.A. 229 du CNRS, Université Pierre et Marie Curie, 4 place Jussieu, F-75252 Paris cedex, France

\begin{abstract}
Experimental (in the 1aboratory and in the field) and theoretical studies indicate that the presence of topographic irregularities can strongly affect (notably increase) the strength of ground motion arising from seismic disturbances. The causes of these effects, particularly as they concern convex topographic features (i.e., hills or mountains) are not well understood, although it is clear that they depend on: the frequency, the precise location on the mountains or in their vicinity (stronger motion at the crests than in the valleys), the aspect ratio of the mountains (stronger for larger aspect ratios) and the angle of incidence (although it is difficult to establish any systematic trends).

In a recent publication [1] we showed that an isolated ridge of rectangular shape responds to an SH wave in the manner of a flanged Helmholtz cavity, with modes of successively higher-order being excited as the frequency increases and the displacement field being maximally amplified at the resonance frequencies for appropriate incident angles at locations on the ridge that are usually on the crest. Indications were provided that the excitation of free modes is the dominant cause of the amplification of ground motion of mountains of this shape. As real mountains are more usually of nearly triangular shape, and are never isolated, it is of substantial interest to determine whether the resonance explanation still holds for the more realistic topographies. We examine herein the $2 \mathrm{D}$ problem of $\mathrm{SH}$ waves striking a group of mutually parallel triangular elastic ridges emerging from flat ground overlying a homogeneous elastic half-space.

Resonances are brought to light in the same manner as in Refs.1, and shown to be excited simultaneously for several orders and over larger bands (than previously), with the result that resonance peaks of displacement are less pronounced and ground amplification is maintained at relatively high levels over wider frequency ranges, in conformity with what is usually observed in terrain measurements. It also turns out that the order of magnitude of the amplification can be predicted by simple geometrical acoustics arguments and that, in many cases, the fields in successive ridges are weakly coupled. The general level of the amplification increases with the aspect ratio of the mountains, but is modest for realistic aspect ratios. The level increases, and approaches that observed in certain experiments if a contrast is introduced in the elastic moduli and densities between the solids occupying the mountains and that of the basement.
\end{abstract}


The mountains are assumed to be infinitely long, mutually parallel ridges emerging from otherwise flat ground; they are subjected to a seismic disturbance in the form of a plane wave (exp(-iwt) implicit time dependence, $\omega$ the angular frequency) with which are associated a propagation vector $\mathbf{k}_{1}$ (pointing upwards) lying in a plane (the sagittal $x-z$ plane $S$ ) perpendicular to the ridge generators and a displacement vector $u$ perpendicular to this plane. The resulting motion is shear horizontal (only $u_{y}$ is non-vanishing) and independent of the coordinate $y$. In the absence of the mountains, the ground is the flat plane $z=0$; the intersection of this plane with $S$ is the line $\mathcal{L}$. The mountains $M_{3}, \quad j \in N(N=\{1,2, \ldots, N\})$ occupy $\Omega_{1}, j \in N$ in $S$ and lie on (in welded contact with) $\mathcal{L}$. The basement $\mathbb{B}(z<0)$ is the half-plane $\Omega_{0}$ in $S$. The junctions of $\Omega_{j}$ with $B$ are the segments $J_{j}$. Each mountain is an isoceles triangle in $S$ with base $J_{j}$ and sides $S_{j}$. The width, height and included angle at the apex of the mountain occupying $\Omega_{j}$ are $w_{j}, b_{j}$ and $\alpha_{j}$ respectively. $B$ and $M_{j}, j \in N$ are filled with linear, elastic, homogeneous, isotropic media $M_{j}, j=0,1,2, \ldots, N$ whose mass density and rigidity are $\rho_{j}$ and $\mu_{j}$ respectively.

Let $u_{i}$ and $u_{j}, j \in N$ be the $y$-components of the (incident and total respectively) displacement vectors $u_{i}$ and $u_{j}$ (in $\Omega_{j}$ ) respectively. $u_{j}$ satisfies: 1) the reduced (Helmholtz) wave equation, 2a) the stress-free boundary condition on $\mathcal{L}-\left(\mathfrak{J}_{1}+\mathfrak{J}_{2}+\ldots+J_{N}\right)$, 2b) the stress-free boundary condition on $\left.S_{j}, j \in N, 3 a\right)$ the continuity of displacement condition on $J_{j}, j \in N, 3 b$ ) the continuity of traction condition on $J_{1}, j \in N$ and 4 ) the Sommerfeld radiation condition (as concerns $u_{0}$ ). The displacement field satisfying 1) in $\Omega_{j} \geqslant 1$ and $2 b$ ) on $S_{j}$ is represented by the (so-called quasi-modal) expansion:

$u_{j}(x)=\sum_{n=0}^{\infty} A_{n}^{j} \frac{J_{n \pi}}{\alpha_{j}}\left(k_{j} r_{j}\right) \cos \left[\frac{n \pi}{\alpha_{j}}\left(\frac{\pi}{2}+\frac{\alpha_{j}}{2}-\psi_{j}\right)\right] ; x \in \Omega_{j} \geqslant 1$.

with $k_{j}=\omega / \beta_{j}, \beta_{j}=\left(\mu_{j} / \rho_{j}\right)^{1 / 2}$ the wavenumber and shear wave velocity in $M_{j}, r_{j}, \psi_{j}$ the polar coordinates with respect to the origin $o_{j}$ located at the apex of $M_{1}$, and $J_{v}(\zeta)$ the $v$-th order Bessel function of argument $\zeta$. The displacement field satisfying 1 ) and 4 ) in $\Omega_{0}$ is represented by:

$u_{0}(x)=u_{i}(x)+u_{r}(x)+\int_{-\infty}^{\infty} B\left(k_{x}\right) \exp \left[i\left(k_{x} x+k_{z} z\right)\right] \frac{d k_{x}}{k_{z}} ; x \in \Omega_{0}$,

with $\left(\theta_{1}\right.$ the angle of incidence with respect to the $+z$ axis $)$ :

$u_{1}(x)=\exp \left[i\left(k_{i x} x-k_{1 z} z\right)\right], k_{i x}=k_{0} \sin \left(\theta_{1}\right), k_{i z}=k_{0} \cos \left(\theta_{i}\right)$,

$u_{r}(x)=\exp \left[i\left(k_{i x} x+k_{1 z} z\right)\right], x=(x, 0, z), k_{z}=\left(k_{0}^{2}-k_{x}^{2}\right)^{1 / 2},\left(\begin{array}{l}\operatorname{Re} \\ \operatorname{Im}\end{array}\right)\left(k_{z}\right) \geqslant 0$

The integral vanishes in the absence of $M_{j} \geqslant_{1}$, in which case the total field in $\Omega_{0}$ reduces to the incident $\left(u_{i}\right)$ plus the reflected $\left(u_{r}\right)$ waves and the modulus of the total displacement (at all points ) on $\mathcal{L}$ to the value 2 . The extent to which this conclusion is modified by the presence of the mountains is analyzed hereafter.

We employ Eqs.(1)-(3) in 2a) and 3b) and project on the Fourier base $\left\{\exp \left(-\mathbf{i} \mathbf{k}_{\mathbf{x}}{ }^{\prime} \mathbf{x} ; \mathbf{k}_{\mathbf{x}}{ }^{\prime} \in \mathbb{R}\right\}\right.$ to obtain:

$B\left(k_{x}\right)=F_{1}\left(A_{n}^{j} ; j \in N, n=0,1,2, \ldots\right) ; k_{x} \in \mathbb{R}$. 
Employing Eqs.(1)-(3) in $3 a)$ and projecting on the base $\left\{\cos \left[\frac{m \pi}{\alpha_{j}}\left(\frac{\pi}{2}+\frac{\alpha_{j}}{2}-\psi_{j}\right)\right]\right.$; $m=0,1,2, \ldots)$ leads to:

$\mathscr{F}_{2 \mathrm{~m}}\left[B\left(\mathrm{k}_{\mathrm{x}}\right) ; \mathrm{k}_{\mathrm{x}} \in \mathbb{R}\right]=\mathscr{F}_{3 \mathrm{~m}}\left(A_{\mathrm{n}}^{J} ; \mathrm{n}=0,1,2, \ldots\right) ; j \in \mathbb{N}, \mathrm{m}=0,1,2, \ldots$

The replacement of $B\left(k_{x}\right)$ in Eq. (5) by its expression given in Eq. (4) finally results in a linear system of equations of the form:

$\sum_{\ell=1}^{N} \sum_{n=0}^{\infty} E_{m n}^{j \ell} A_{n}^{\ell}=G_{m}^{j} \quad ; j \in N, m=0,1,2, \ldots$

the only term of which involving the incident wave parameters is $G_{m}^{j}$. This infinite-order linear system is solved by truncation and the process is repeated as many times (with increasing truncation order) as is necessary to obtain stabilization of the numerical values of the most significant coefficients $A_{n}^{\ell}$. The latter are then introduced into Eq. (5) to obtain the plane-wave amplitudes $B\left(k_{x}\right)$ and into Eqs. (1) and (2) to compute the displacement fields.

Let us restrict the discussion to the surface field on a representative mountain. For the amplification to be a phenomenon of systematic nature, such as is generally observed (this meaning that it can occur practically at any location of the mountain surface and for essentially any incident angle), one or more of the coefficients $A, l$ must be large; for this to occur independently of the incident angle the determinant of the hypermatrix $\mathbb{E}$ in Eq. (6) must be small, at least for certain frequencies. The latter are none other than the eigenfrequencies, because the singularity of $\mathbb{E}$ is equivalent to the existence of a natural (eigen) mode, $\operatorname{det}(\mathbb{E})=0$ giving rise to the dispersion relations of the eigenmodes of the structure, and to a resonance which occurs when the driving frequency is equal to the (real part of) one of the eigenfrequencies. The spectral response is lorentzian in the neighborhood of the eigenfrequencies and finite due to radiation damping. From Eq.(6) it is possible to demonstrate that: a) each $(n, j)$-th quasi-mode becomes large at its own set of frequencies $\omega(j, n, m)$ ; $m=0,1,2, \ldots$, with $m=0$ the fundamental and $m \geqslant 1$ the overtones, $b$ ) for a set of identical mountains, the $(j, n)$ eigenfrequencies are degenerate with respect to $j$ and splitting occurs with the appearance of a set of resonant frequencies above and below the resonance frequencies of the isolated mountains.

To illusrate these predictions, consider Fig.1 which applies to a single-mountain configuration. At the apex the field involves only the lowest-order quasi mode, i.e., $\left|u_{1}\right|=\left|A{ }_{0}^{1}\right|$. The observed peaks are therefore located at the resonance frequencies $\omega(1,0,0), \omega(1,0,1), \ldots$ and these frequencies are close to the real parts of the roots of $\operatorname{det}\left(\mathbb{E}^{\prime}\right)=0$, with $\mathbb{E}^{*}=$ $\left\{\mathrm{E} \begin{array}{l}11 \\ 0\end{array}\right\}$. Since the successive resonance bands are rather wide and close together, overlapping occurs with the result that the overall level is relatively high, even at the frequencies furthest from the center frequencies of the resonance bands. The figure also reveals that the amplification is rather stable and small for the most realistic mountain shapes $\left(\alpha=90,120^{\circ}\right)$; however, a slower, less rigid material within the mountain accentuates the resonances (due to better energy trapping) and thereby increases substantially the peak amplifications. The horizontal line at level 2 in Fig.1 represents the geometrical acoustics (GA) prediction of the apex response of the $\alpha=90^{\circ}$ mountain. It can be observed that this response constitutes a rather good approximation of the over-all response at all except the lowest frequencies. In the case the media filling $M_{1}$ and $B$ are identical the GA predicts an apex amplification of $360 / \alpha\left({ }^{\circ}\right)$, in good agreement with what is observed in the figure.

The effect of neighbors can be ascertained in Fig.2, where the displacement 
field is now sampled at a point half-way up the left side of the left-hand mountain of one and two-mountain configurations. Since the plane wave strikes the mountains at normal $\left(\theta_{1}=0^{\circ}\right)$ incidence, only the even-order quasi-modes are excited, but all of the latter contribute to the field on the side of the mountain. Therefore, the response is both the result of order-to-order mode mixing and overtone-to-overtone mixing with the result that the amplifications are smaller than at the apex. Splitting is readily observed, with the largest effects occuring at low frequencies. At higher frequencies, the two-mountain response closely follows the one-mountain response, indicating rather small coupling effects.

[1] L. Kouoh-Bille, F.J. Sanchez-Sesma and A. Wirgin, C.R.Acad.Sci. II $312(1991) 849$.

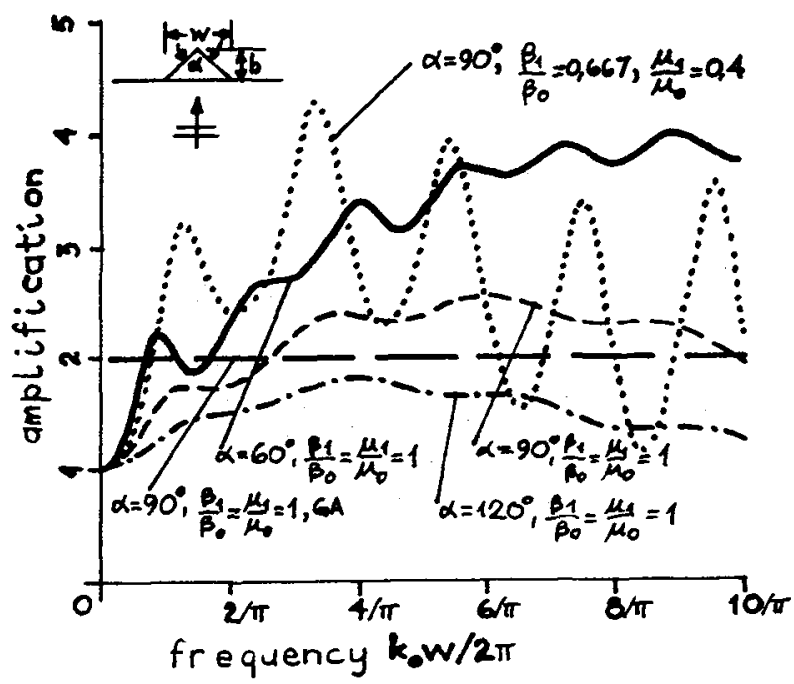

Fig. 1 Amplification $\left|u_{1}\right| / 2$ versus frequency at the apex for a single mountain.

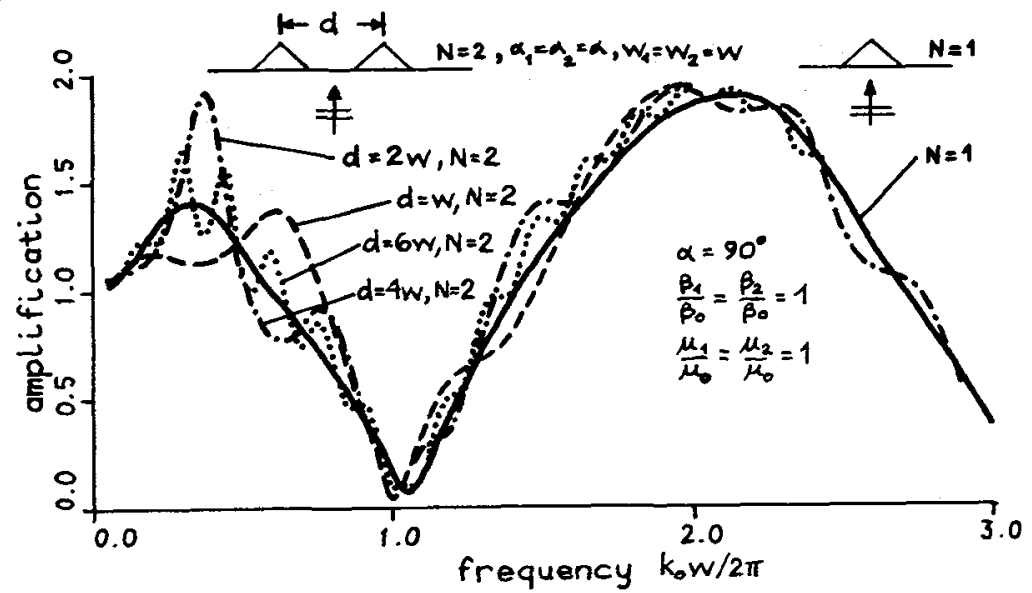

Fig. 2 Amplification $\left|u_{1}\right| / 2$ versus frequency at mid-helght on a mountain of one$(N=1)$ and two- $(\mathrm{N}=2)$ mountain configurations. 